\title{
Targeting of Non-Structural Protein 9 as a Novel Therapeutic Target for the Treatment of SARS-CoV-2
}

\author{
Matthew P. Anderson'1, John J. Turchi'2,3 \\ ${ }^{1}$ Indiana University School of Medicine, Indiana University School of Medicine, ${ }^{2}$ Department of \\ Medicine and ${ }^{3}$ Department of Biochemistry and Molecular Biology
}

\section{Background/Hypothesis:}

The 2019 novel coronavirus (SARS-CoV-2) is a human coronavirus responsible for a global pandemic with over 13 million confirmed cases. Currently, there are no treatments to block viral infection or replication. Exploring novel therapeutic targets for SARS-CoV-2 and future coronaviruses holds great promise for treating the current and future outbreaks. One such target is the non-structural protein 9 (nsp9), which has been shown to be highly conserved and unique to the coronavirus family as well as playing a role in viral replication. We hypothesize nsp9 is a viable target for therapeutic development.

\section{Methods:}

Towards determining the utility of targeting nsp9, a series of databases were queried for articles pertaining to nsp9 in SARS-CoV-2 and other coronaviruses and coronaviruses in general. We assessed structural, biochemical and cellular features of nsp9.

\section{Results:}

Nsp9 forms a homodimer via a conserved $\alpha$-helix containing a glycine-rich interaction motif (GxxxG). Dimerization at the GxxxG interface is required for efficient viral replication. Nsp9's core is an open, six-stranded $\beta$-barrel whose fold gives it a structure similar to nucleic acid binding OB-fold proteins. This OB-like fold has not been detected in replicative complexes of other RNA viruses and may reflect the unique and complex CoV replication machinery. Nsp9 is an indispensable component of the replication complex that binds single-stranded RNA in a concentration-dependent manner. A recent bioinformatic approach also found that nsp9 interacts with NF-kappa-B-repressing factor and may play a role in the IL-8/IL-6 mediated chemotaxis of neutrophils and inflammatory response observed in Covid-19 patients.

\section{Conclusion/Potential Impact:}

Based on this research, we conclude nsp9 represents a novel therapeutic target whose OB-likefold may provide a targetable structure for interrupting RNA binding and impairing viral replication. This study will help inform current and future research that seeks to target nsp9's structure and biochemical interactions as treatment for coronavirus infection. 